05

\title{
Моделирование динамического старта трещины на основе перидинамической численной модели и критерия инкубационного времени
}

\author{
(C) М.О. Игнатьев, ${ }^{1}$ Ю.В. Петров, ${ }^{1,2}$ Н.А. Казаринов ${ }^{1,3}$ \\ ${ }^{1}$ Санкт-Петербургскии государственный университет, \\ 199034 Санкт-Петербург, Россия \\ ${ }^{2}$ Институт проблем машиноведения РАН, \\ 199178 Санкт-Петербург, Россия \\ ${ }^{3}$ Петербургский государственный университет путей сообщения Императора Александра I, \\ 190031, Санкт-Петербург, Россия \\ e-mail: ignatiev_maksim@mail.ru
}

Поступило в Редакцию 8 июня 2020 г.

В окончательной редакции 1 августа 2020 г.

Принято к публикации 11 сентября 2020 г.

Исследованы вопросы динамического хрупкого разрушения в условиях высокоскоростного нагружения. Новая численная схема на основе перидинамического подхода и структурно-временной модели разрушения применяется для прогнозирования старта трещины в образцах из стеклообразного полимерного материала. Приведено сравнение результатов расчетов с экспериментальными данными.

Ключевые слова: динамика, прочность, разрушение, трещины, перидинамика, критерий разрушения, инкубационное время.

DOI: $10.21883 /$ JTF.2021.03.50520.196-20

\section{Введение}

В классических экспериментальных работах [1,2], посвященных вопросам старта, распространения и остановки трещин в условиях ударно-волнового воздействия, был изучен ряд принципиальных фундаментальных эффектов динамической механики разрушения. В частности, в работе [1] представлена измеренная зависимость стартового значения коэффициента интенсивности напряжений (КИН) от времени инициации движения трещины. Эксперименты, проведенные упомянутыми авторами над образцами фотоупругого материала Гомалит-100 (Homalite-100), продемонстрировали заметную скоростную (временную) зависимость КИН в момент старта трещины: в случае высокоскоростного нагружения стартовые значения КИН значительно превышали критическое значение статического коэффициента интенсивности данного материала. Таким образом, было показано, что даже для оценки разрушения в образцах с неподвижными трещинами использование общепринятого для статических задач энергетического подхода Ирвина-Гриффитса [3] не представляется возможным. Традиционно данная проблема решается прямым обобщением критерия Ирвина на случай динамического нагружения (внедрение динамических аналогов статических критических параметров, например, параметра $K_{I D}$ - ударной вязкости материала), заменой критических прочностных констант материала на сложные функционалы (в частности, использование зависимости текущего значения КИН от скорости трещины в каче- стве характеристики материала, определяющей процесс распространения трещины [4]) или вводом в модель материала явной зависимости прочности от скорости деформации (одной из самых распространенных моделей такого типа - модель Джонсона-Кука [5]). Несмотря на свою распространенность, такие подходы и модели зачастую опираются на параметры и зависимости, которые затруднительно отнести к свойствам материала и которые нуждаются в переопределении для каждой конкретной задачи.

В работе [6] на основе перидинамического подхода был смоделирован динамический старт роста трещины с использованием классического критерия разрушения Ирвина-Гриффитса. При временах до разрушения, близких к нулю (менее $2.5 \mu \mathrm{s}$ ), модель [6] предполагает резкое увеличение критического КИН, но при этом авторами не приводится ни одного сравнения численных результатов с какими-либо экспериментально полученными зависимостями критического КИН от времени разрушения. В то же время ввиду вышесказанного использование классического критерия разрушения для моделирования динамической инициации роста трещины представляется сомнительным.

В $[7,8]$ был предложен структурно-временной подход к прогнозированию динамического разрушения материалов, основанный на использовании характерного размера зоны разрушения материала, а также характерного времени релаксации материала при разрушении - инкубационного времени. Структурно-временная модель разрушения была успешно применена для широкого спек- 
тра задач динамической механики разрушения [9-11]. Ниже исследуется возможность применения структурновременного подхода для прогнозирования эффектов процессов разрушения у вершины трещины на основе перидинамического моделирования.

\section{1. Численная схема}

\section{1. Перидинамический подход к задачам механики сплошной среды}

Перидинамика - численный подход к задачам сплошной среды, в соответствии с которым твердое тело представляется в виде совокупности материальных частиц, осуществляющих нелокальное взаимодействие между собой $[12,13]$. В перидинамическом теле поведение каждой частицы $\mathbf{x}$, которая занимает объем $d V_{\mathbf{x}}$, определяется ее взаимодействием с соседними частицами $\mathbf{x}^{\prime}$, которые находятся в сферической окрестности (соседства) $\mathscr{H}_{x}$ радиуса $\delta$ (численно стабильным является выбор $\delta=3 \Delta x$, где $\Delta x-$ расстояние между частицами в отсчетной конфигурации [14]). Таким образом, устанавливается принципиально нелокальный характер взаимодействия частиц тела. Классическая перидинамическая формулировка законов движения была предложена в [12]:

$$
\rho(\mathbf{x}) \ddot{\mathbf{u}}(\mathbf{x}, t)=\int_{\mathscr{G}_{x}} \mathbf{f}\left(\mathbf{x}^{\prime}, \mathbf{x}, t\right) d V_{\mathbf{x}^{\prime}}+\mathbf{b}(\mathbf{x}, t),
$$

где $\mathbf{f}\left(\mathbf{x}^{\prime}, \mathbf{x}, t\right)$ - функция попарного взаимодействия частиц (плотность силы связи, размерность которой сила/объем $\left.{ }^{2}\right)$, определяющая реологию моделируемого материала; $\rho(\mathbf{x}), \ddot{\mathbf{u}}(\mathbf{x}, t), \mathbf{b}(\mathbf{x}, t)$ - поля плотности, ускорений и объемных сил соответственно.

В отличие от классических уравнений равновесия механики сплошной среды уравнение (1) не содержит операторов дифференцирования по координате, что делает перидинамический подход эффективным для задач с сингулярными полями, в частности, для задач механики разрушения. Однако относительно простая классическая формулировка (1) (в которой силовой вектор связи частицы $\mathbf{x}$ с частицей $\mathbf{x}^{\prime}$ не зависит от деформаций связей с другими частицами из соседства $\mathscr{H}_{x}$ ) накладывает и существенное ограничение на механические свойства моделируемого материала: эффективный коэффициент Пуассона такого материала в трехмерном случае всегда равен 0.25 [12]. Обобщение перидинамической модели, позволяющее обойти данную особенность, было проведено в работе [13]. В переформулированной теории попарное взаимодействие частиц заменено более сложным функционалом, основанном на понятии векторного состояния. Векторное состояние, подобно тензору второго ранга, может сопоставлять одному вектору некий другой вектор, однако в отличие от тензора векторное состояние может быть нелинейной и прерывной функцией вектора связи $\mathbf{x}^{\prime}-\mathbf{x}$.
Силовое состояние $\underline{\mathbf{T}}$, определенное в $\mathbf{x}$ и в момент времени $t$, сопоставляет каждой связи $\mathbf{x}^{\prime}-\mathbf{x}$ из $\mathscr{H}_{x}$ некоторый силовой вектор $\underline{\mathbf{T}}[\mathbf{x}, t]\left\langle\mathbf{x}^{\prime}-\mathbf{x}\right\rangle$. Таким образом, уравнения равновесия обобщенной перидинамической теории могут быть записаны следующим образом:

$$
\begin{aligned}
\rho(\mathbf{x}) \ddot{\mathbf{u}}(\mathbf{x}, t)= & \int_{\mathscr{H}_{x}}\left\{\underline{\mathbf{T}}[\mathbf{x}, t]\left\langle\mathbf{x}^{\prime}-\mathbf{x}\right\rangle\right. \\
& \left.-\underline{\mathbf{T}}\left[\mathbf{x}^{\prime}, t\right]\left\langle\mathbf{x}-\mathbf{x}^{\prime}\right\rangle\right\} d V_{\mathbf{x}^{\prime}}+\mathbf{b}(\mathbf{x}, t) .
\end{aligned}
$$

Перидинамический подход подразумевает, как правило, численные решения уравнений (2). В настоящей работе для вычислений использовался программный комплекс Peridigm, разработанный организацией Национальные лаборатории Сандия [15]. Была использована ординарная линейная упругая модель материала (Linear peridynamic solid, [13]). Для расчета КИН вычислялся динамический Ј-интеграл согласно формуле (3.1b) из [16].

Предполагается, что разрыв связей происходит независимо друг от друга при выполнении определенного критерия. Один из распространенных подходов основан на задании критериального условия в виде критического растяжения связи $[14,17,18]$, являющегося перидинамическим аналогом критерия Гриффитса. В настоящей работе в качестве условия разрушения связи применяется структурно-временной критерий [7-9], основанный на понятия инкубационного времени.

\section{2. Критерий инкубационного времени разрушения}

Согласно структурно-временному подходу [7-9,19], макроскопическое разрушение имеет место как следствие некоторых подготовительных процессов (например, развитие и слияние микротрещин, пор, дефектов). Предполагается, что данные процессы развиваются под воздействием нормальных напряжений в течение характерного для данного материала времени - инкубационного времени. Таким образом, согласно данному подходу, разрушение не происходит мгновенно. В рамках настоящей работы предлагается использовать подход инкубационного времени к описанию разрушения перидинамических связей. Условие разрушения связи в момент $t^{*}$ запишем в виде интегрального соотношения:

$$
\frac{1}{\tau} \int_{t^{*}-\tau}^{t^{*}} \sigma^{\text {bond }}\left(t^{\prime}\right) d t^{\prime} \geq \sigma_{c}^{\text {bond }}
$$

где $\tau$ - инкубационное время, $\sigma^{\text {bond }}(t)-$ действующие в связи нормальные напряжения, $\sigma_{c}^{\text {bond }}-$ критическое статическое напряжение рассматриваемой связи. В настоящей работе критерий (3) применялся для предсказания разрушения связи, а в качестве критического статического напряжения использовалось критическое 
напряжение связи $\sigma_{c}^{\text {bond }}$, зависящее от размера перидинамического элемента. Значение данного параметра выбиралось исходя из соответствия критерия (3) критерию Гриффитса для квазистатической задачи об инициации и распространении трещины.

\section{2. Результаты}

В настоящей работе исследовалась задача о росте симметрично нагружаемой трещины, вызываемом ударноволновым равномерным воздействием на ее берега в соответствии с экспериментальной схемой, реализованной в $[1,2]$. В данных исследованиях изучались старт, продвижение и остановка трещины в материале Гомалит-100. Одним из результатов была экспериментальная зависимость стартового значения КИН от времени разрушения (момента старта трещины). В экспериментах испытывались прямоугольные пластины с предварительным боковым разрезом. Ударное давление на берега разреза, имитирующего трещину, имело временной профиль в форме трапециевидного импульса с длительностью участка линейного нарастания давления до амплитудного значения - $25 \mu \mathrm{s}$ (рассматривался диапазон амплитуд: $0.8-12 \mathrm{MPa})$.

Разработанная численная схема на основе перидинамического подхода и критерия (3) была применена для

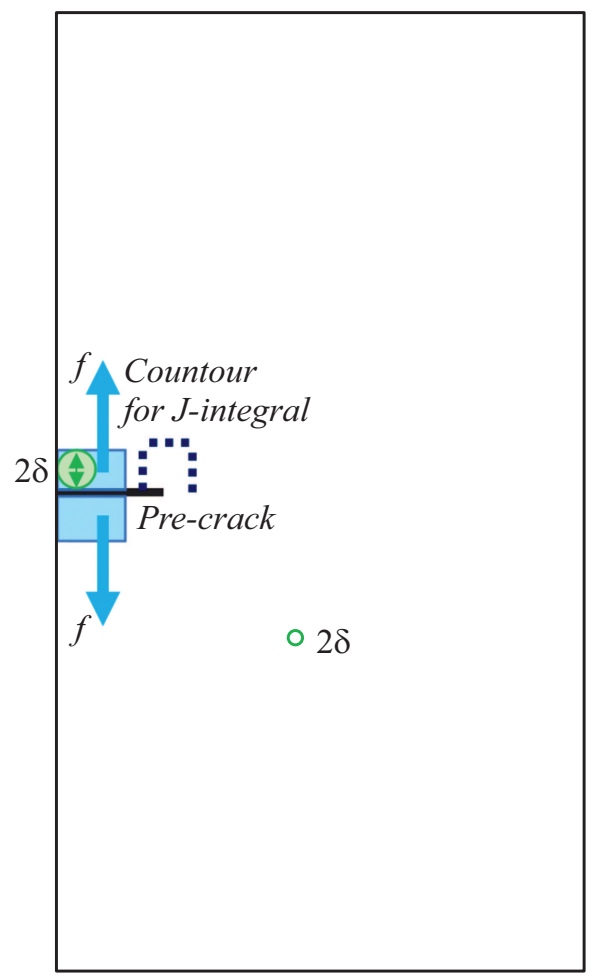

Рис. 1. Схема проведения расчета: $f-$ сила, приложенная к нелокальной области шириной $2 \delta$; толщина образца была выбрана также $2 \delta$, поскольку Peridigm реализован только для трехмерного моделирования. Пунктирной линией обозначен контур, по которому вычислялся динамический $J$-интеграл.

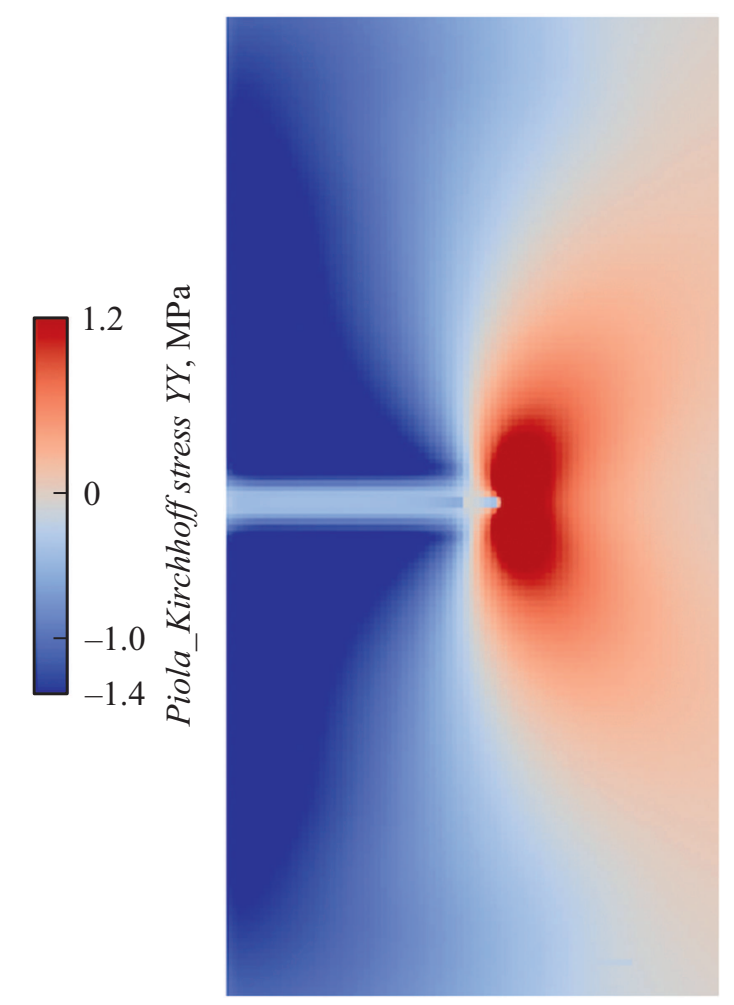

Рис. 2. Поле нормальных напряжений в направлении приложенной вертикальной нагрузки (изображена часть пластины, содержащая трещину).

определения момента старта трещины и вычисления стартового значения КИН. При моделировании применялись следующие параметры:

\begin{tabular}{l|l}
\hline Модуль Юнга, $E$ & $3.9 \mathrm{GPa}$ \\
\hline Коэффициент Пуассона, $v$ & 0.35 \\
\hline $\begin{array}{l}\text { Критическое напряжение связи (для } \\
\text { использованного разбиения), } \sigma_{c}^{\text {bond }}\end{array}$ & $8.57 \mathrm{MPa}$ \\
\hline Инкубационное время, $\tau$ & $9 \mu \mathrm{s}$
\end{tabular}

Значение инкубационного времени $\tau=9 \mu$ s было получено при аналитическом решении аналогичной задачи в работе [19]. Моделирование экспериментов проводилось при помощи разработанной расчетной схемы на основе перидинамического подхода и критерия инкубационного времени. Была использована явная численная схема, перидинамический элемент имел форму куба с размером ребра $\Delta x=0.5 \mathrm{~mm}$, радиус сферического соседства $\delta=1.5 \mathrm{~mm}$, а временной шаг расчета составил $0.18 \mu \mathrm{s}$. Размер образца был $150 \times 250 \times 3 \mathrm{~mm}$ с предварительным разрезом длиною $25 \mathrm{~mm}$. Размер был выбран таким, чтобы в течение расчета отраженные от границ образца волны не успевали достичь вершины трещины. Вид нагружения (рис. 1), а также 


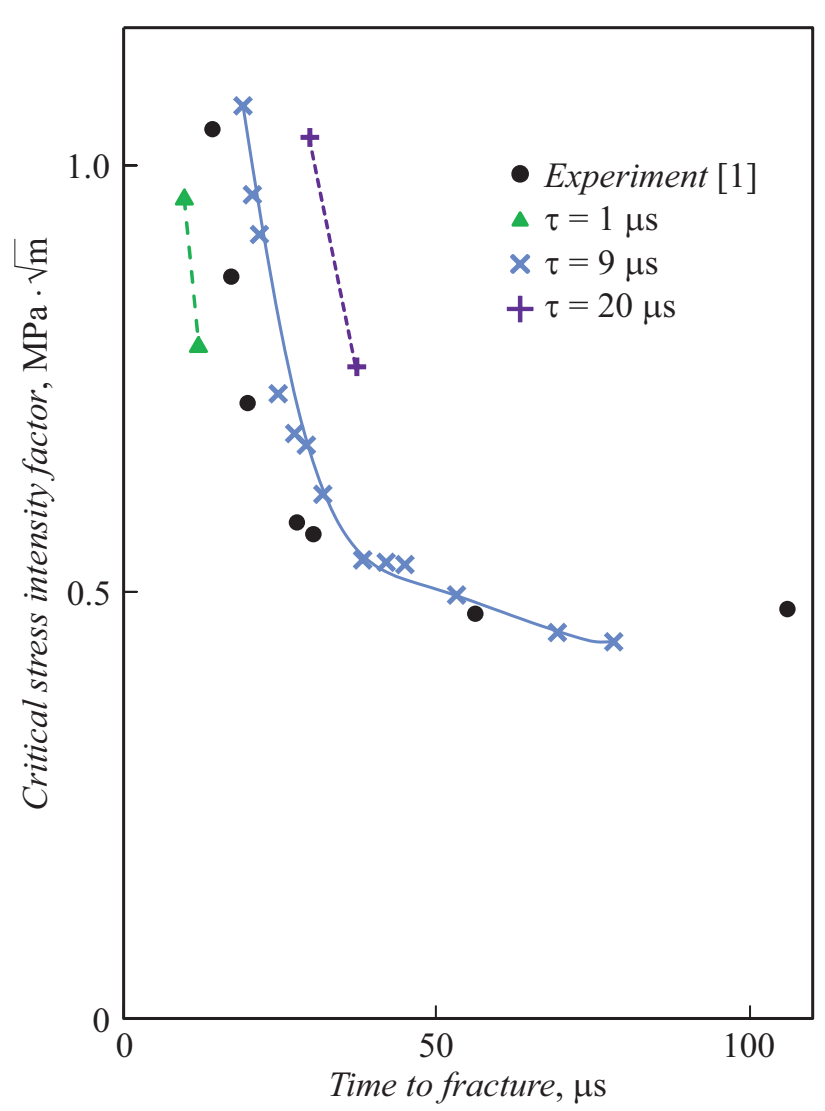

Рис. 3. Зависимость порогового КИН в момент инициации трещины от времени до разрушения $t^{*}$. Кружками показаны экспериментальные данные [1], пунктирными кривыми результаты расчета методом перидинамики для различных значений $\tau$.

свойства материала были аналогичны параметрам из работ $[1,2]$. На рис. 2 изображено распределение поля напряжений $\left(\sigma_{y y}\right)$ до момента инициации роста трещины. Результаты расчета инициации роста трещины для различных значений параметра инкубационного времени представлены на рис. 3. Каждой точке на приведенных кривых (как расчетных, так и экспериментальных) соответствует эксперимент с определенным значением амплитуды нагружающего импульса. Было показано, что увеличение параметра $\tau$ приводит к росту стартового значения КИН, и наоборот, относительно малым значениям инкубационного времени соответствует старт трещины при низких значениях КИН, близких к квазистатическому.

Показано, что для значения $\tau=9 \mu$ s полученная кривая согласуется с экспериментальными данными как при малых временах до разрушения (большие амплитуды воздействия, динамическая ветвь), так и в квазистатическом случае $\left(t^{*} \geq 50 \mu \mathrm{s}\right.$, статическая ветвь), что говорит об эффективности структурно-временного подхода в соединении с перидинамической численной схемой для прогнозирования динамической трещиностойкости твердых тел.

\section{Заключение}

В работе представлена расчетная схема на основе перидинамического подхода и структурно-временного критерия, основанного на идее инкубационного времени разрушения. Исследовалась задача инициации трещины в условиях высокоскоростного ударно-волнового воздействия. Также важным результатом является перидинамическая формулировка инкубационного критерия в терминах разрушения связи. Сочетание нелокального перидинамического подхода к вычислению напряжений, а также учет инкубационных характеристик процесса разрушения вкупе с локальной историей напряжений при помощи инкубационного времени позволили достаточно точно описать наблюдаемые экспериментально зависимости стартового значения КИН от времени старта трещины. Моделирование подобных зависимостей при помощи классических прочностных критериев (например, на основе статических и динамических критических значений КИН) невозможно, в то же время представленный в работе подход представляется перспективным для эффективной оценки прочности конструкций в режимах высокоскоростного динамического воздействия.

\section{Благодарности}

Авторы выражают благодарность профессору университета Стратклайда и главе Центра исследований по перидинамике г. Глазго (Великобритания) доктору Э. Отеркусу (Dr. E. Oterkus) за поддержку при разработке примененной в работе численной перидинамической модели, а также И.В. Михновичу за содействие в написании программного кода.

\section{Финансирование работы}

Работа выполнена при поддержке РФФИ (№ 20-01-00291, № 19-31-60037). Разд. 1 и 3 выполнены Ю.В. Петровым в рамках проекта РНФ (№ 17-11-01053).

\section{Конфликт интересов}

Авторы заявляют, что у них нет конфликта интересов.

\section{Список литературы}

[1] K. Ravi-Chandar, W.G. Knauss. Intern. J. Fracture, 25, 247 (1984). DOI: 10.1007/BF00963460

[2] K. Ravi-Chandar, W.G. Knauss. Intern. J. Fracture, 26, 141 (1984). DOI: 10.1007/BF01157550

[3] G. Irwin. J. Appl. Mechan., 24, 361 (1957).

[4] A.J. Rosakis, G.J. Ravichandran. Mech. Mater. Struct., 37, 331 (2000). DOI: 10.1016/S0020-7683(99)00097-9

[5] G. Johnson, W. Cook. Eng. Fract. Mech., 21 (1), 31 (1985). DOI: $10.1016 / 0013-7944(85) 90052-9$

[6] R. Panchadhara, P.A. Gordon. Intern. J. Fracture, 201 (1), 81 (2016). DOI: $10.1007 / \mathrm{s} 10704-016-0124-8$ 
[7] Y.V. Petrov, A.A. Utkin. Sov. Mater. Sci., 25 (2), 153 (1989). DOI: $10.1007 / \mathrm{BF} 00780499$

[8] Ю.В. Петров. ДАН, 321, 66 (1991). [Y.V. Petrov. Dokl Akad Nauk SSSR, 321 (1), 66 (1991).]

[9] Ю.В. Петров, Е.В. Ситникова. ЖТФ, 74 (1), 58 (2004). [Y.V. Petrov, E.V. Sitnikova. Tech. Phys., 49, 57 (2004). DOI: $10.1134 / 1.1642679]$

[10] Y.V. Petrov, B.L. Karihaloo, V.V. Bratov, A.M. Bragov. Intern. J. Engineer. Sci., 61, 3 (2009). DOI: 10.1016/j.ijengsci.2012.06.004.

[11] V.A. Bratov, N.A. Kazarinov, Y.V. Petrov. J. Physics: Conf. Series, 653 (1), 012050 (2015).

DOI: $10.1088 / 1742-6596 / 653 / 1 / 012050$

[12] S.A. Silling. J. Mechan. Phys. Solid., 48(1), 175 (2000). DOI: 10.1016/S0022-5096(99)00029-0

[13] S.A. Silling, M. Epton, O. Weckner, J. Xu, E. Askari. J. Elasticity, 88, 151 (2007). DOI: 10.1007/s10659-007-9125-1

[14] Y.D. Ha, F. Bobaru. Int. J. Fract., $162(1-2), 229$ (2010). DOI: $10.1007 / \mathrm{s} 10704-010-9442-4$

[15] M.L. Parks, D.J. Littlewood, J.A. Mitchell, S.A. Silling. Peridigm Users' Guide, Tech. Report SAND2012-7800 (Sandia National Laboratories, 2012).

[16] T. Nakamura, C.F. Shih, L.B. Freund. Intern. J. Fracture, 27, 229 (1985). DOI: 10.1007/BF00017970

[17] E. Madenci, E. Oterkus. Peridynamic Theory and Its Applications (Springer, NY., 2014).

[18] F. Bobaru, T.J. Foster, P.H. Geubelle, S.A. Silling. Handbook of peridynamic modeling (CRC press., 2016).

[19] Y.V. Petrov, N.F. Morozov. J. Appl. Mech., 61, 710 (1994). DOI: $10.1115 / 1.2901518$ 